\title{
A GASTROPLASTIA EM MANGA (SLEEVE GASTRECTOMY) E O DIABETES MELLITUS
}

\author{
Sleeve gastrectomy and diabetes mellitus
}

\author{
Alexandre Ciro ANDRIANI, Thais Torres NEVES
}

ABCDDV/610

Andriani AC, Neves TT. A gastroplastia em manga e o diabetes mellitus. ABCD Arq Bras Cir Dig 2008;21(3):133-5

RESUMO - Racional - O tipo 2 da diabete mellitus é muito frequente entre os pacientes de obesidade morbida. Ja foi provado cientificamente que o bypass gástrico pode curá-lo. Existem poucos relatos de que a gastroplastia em manga apresenta resultado promissores na cura desta forma de diabete. Objetivo - Apresentar resultados da glicemia em pacientes operados com a gastroplastia em manga mostrando sua possibilidade de controlar o dibetes. Método - Um estudo prospectivo envolvendo 15 pacientes foi realizado nos quais as dosagens sericas da glicose foram medidas no pré e pós-operatório. Para que houvesse homogeneidade da amostra, critérios de inclusão e exclusão foram estabelecidos com base nos pacientes com IMC de 35 a 40. Todos os pacientes foram submetidos a técnica cirurgica de gastroplastia em manga. O seguimento se deu em atendimento ambulatorial. Resultados - Todos os pacientes foram operados pela técnica referida e seus níveis glicêmicos computados no pré, pós-operatório e no seguimento. Conclusão - A gastroplastia em manga cura o diabetes mellitus mostrando queda logo após a operação e mantendo-a ao longo do seguimento. DESCRITORES - Gastrectomia. Diabetes mellitus. Obesidade mórbida.

\section{INTRODUÇÃO}

O diabetes mellitus (T2DM) tem alta prevalência nos pacientes obesos mórbidos, com consequências bastante graves. Já foi demonstrado em diversos trabalhos que as gastroplastias com desvio do trânsito duodenal cursam com melhora e até sua cura, antes mesmo que haja emagrecimento substancial dos pacientes operados que justifique essa melhora glicêmica. A gastroplastia vertical com derivação jejuno-ileal é conhecida como uma operação mista com predominância do componente restritivo sobre o disabsortivo, e nessa modalidade a reversão do T2DM teoricamente deve-se ao aumento da sensibilidade à insulina associado à melhora da função da célula beta.

O primeiro trabalho a enfocar a prevenção do diabetes foi realizado por Long et al. ${ }^{10}$, que observaram uma queda no risco relativo de desenvolvimento do diabetes mellitus de 30 vezes em obesos mórbidos operados quando comparados aos não operados ${ }^{10}$. Uma das primeiras grandes séries de estudo de operações em diabéticos é o estudo de Greenville (EUA), no qual 165 pacientes diabéticos foram submetidos ao bypass gástrico e $83 \%$ destes permaneceram com remissão do diabetes em 14 anos de seguimento ${ }^{11}$. Um grande trabalho prospectivo e em andamento é o SOS (Swedish Obesity Subjects), que compara um grupo de

\footnotetext{
Trabalho realizado no Serviço de Cirurgia Geral do Hospital Regional de São José Homero de Miranda Gomes, São José, SC, Brasil.

Correspondência: alexandre.andriani@gmail.com
}

pacientes operados com um outro não operado, que poderá esclarecer ainda alguns pontos.

Esta estudo tem por objetivo avaliar os resultados da glicemia no pós-operatório de cirurgia bariátrica.

\section{MÉTODOS}

Foram avaliados prospectivamente os pacientes submetidos à gastroplastia em manga (sleeve gastrectomy SG) no Hospital Regional de São José Homero de Miranda Gomes (HRSJHMG), São José, SC, Brasil, no período de janeiro a novembro de 2008 que apresentavam diagnóstico prévio de diabetes mellitus ou intolerância à glicose. Os dados foram colhidos nas consultas quinzenais de rotina após a operação no ambulatório geral deste hospital, tendo sido acompanhado nos três primeiros meses quanto às alterações nas glicemias.

Os resultados foram tabulados eletronicamente, constando o número de registro hospitalar, sexo, idade, data da operação e o valor da glicemia. Como a perda de peso nos três primeiros meses não é suficiente por si só para justificar melhora nas glicemias, este período foi considerado o mais importante e foi o estudado neste trabalho.

Critérios de inclusão: pacientes operados no HRSJHMG pela técnica SG com índice de massa corporal (IMC) maior ou igual $35 \mathrm{~kg} / \mathrm{m}^{2}$ com diabetes mellitus ou IMC maior que 40 e intolerância à glicose, e que aceitaram participar desta pesquisa e assinaram espontaneamente o 
termo de consentimento informado.

Critérios de exclusão: pacientes que foram operados por outras técnicas que não a $S G$ ou que não apresentavam hiperglicemia nos exames realizados no pré-operatório. $\mathrm{O}$ número da amostragem foi de 15 pacientes que satisfizeram todos os quesitos.

\section{RESULTADOS}

Todos os 15 pacientes submetidos à técnica de SG obtiveram redução dos seus níveis glicêmicos em um pós-operatório precoce e alguns atingiram a faixa da normalidade da taxa glicêmica logo nos primeiros dias. Dos $100 \%$ operados pela técnica de $\mathrm{SG}, 53,33 \%(\mathrm{~N}=8$ pacientes) obtiveram cura total do T2DM no seguimento da pesquisa; e $46,66 \%$ (N=7 pacientes) atingiram diminuição dos seus níveis glicêmicos do pré-operatório, e destes últimos $57,14 \%$ não necessitam mais do uso de insulina. Ressalte-se que foram computados apenas os três primeiros meses após a cirurgia, sendo descartados os meses seguintes (Tabela 1).

TABELA 1 - Dosagens glicêmicas no pré e pós-operatório

\begin{tabular}{cll}
\hline $\begin{array}{c}\text { PACIENTES DO } \\
\text { ESTUDO }\end{array}$ & $\begin{array}{l}\text { GLICEMIA } \\
\text { PRÉ-OPERATÓRIO }\end{array}$ & $\begin{array}{l}\text { GLICEMIA } \\
\text { PÓS -OPERATÓRIO }\end{array}$ \\
\hline 1 & $136,3 \mathrm{mg} / \mathrm{dl}$ & $98,6 \mathrm{mg} / \mathrm{dl}$ \\
2 & $123 \mathrm{mg} / \mathrm{dl}$ & $82,1 \mathrm{mg} / \mathrm{dl}$ \\
3 & $168 \mathrm{mg} / \mathrm{dl}$ & $133 \mathrm{mg} / \mathrm{dl}$ \\
4 & $126 \mathrm{mg} / \mathrm{dl}$ & $115 \mathrm{mg} / \mathrm{dl}$ \\
5 & $131 \mathrm{mg} / \mathrm{dl}$ & $97 \mathrm{mg} / \mathrm{dl}$ \\
6 & $145 \mathrm{mg} / \mathrm{dl}$ & $88 \mathrm{mg} / \mathrm{dl}$ \\
7 & $175,3 \mathrm{mg} / \mathrm{dl}$ & $127,2 \mathrm{mg} / \mathrm{dl}$ \\
8 & $203 \mathrm{mg} / \mathrm{dl}$ & $145 \mathrm{mg} / \mathrm{dl}$ \\
9 & $127,9 \mathrm{mg} / \mathrm{dl}$ & $73 \mathrm{mg} / \mathrm{dl}$ \\
10 & $198 \mathrm{mg} / \mathrm{dl}$ & $145 \mathrm{mg} / \mathrm{dl}$ \\
11 & $152 \mathrm{mg} / \mathrm{dl}$ & $99,2 \mathrm{mg} / \mathrm{dl}$ \\
12 & $167 \mathrm{mg} / \mathrm{dl}$ & $110 \mathrm{mg} / \mathrm{dl}$ \\
13 & $141 \mathrm{mg} / \mathrm{dl}$ & $76 \mathrm{mg} / \mathrm{dl}$ \\
14 & $203,9 \mathrm{mg} / \mathrm{dl}$ & $178 \mathrm{mg} / \mathrm{dl}$ \\
15 & $160,4 \mathrm{mg} / \mathrm{dl}$ & $122 \mathrm{mg} / \mathrm{dl}$ \\
\hline
\end{tabular}

\section{DISCUSSÃO}

A prevalência da obesidade mórbida continua aumentando rapidamente no mundo. Ela está fortemente relacionada com o T2DM, o que a torna um problema grave de saúde pública.

Nos EUA a prevalência vem aumentando em 198\%, e de 16.2 milhões em 2005 passará, segundo as previsões, para 48.3 milhões em $2050^{10}$.

Para o tratamento cirúrgico da obesidade mórbida em associação com o T2DM várias técnicas têm sido empregadas, entre os quais a mais frequente é a gastroplastia de Fobi-Capella, que já apresenta grandes casuísticas ${ }^{11}$.

A SG tem poucos relatos na literatura da sua aplicação para cura da obesidade mórbida e controle de comorbidades, e apresenta apenas um relato de sua eficácia na cura do T2DM, com resultados promissores ${ }^{14}$.

Nas outras técnicas, a cura, remissão ou controle sem medicamentos do T2DM é atingido em até $80 \%$ dos casos, sendo que a remissão pode ocorrer em $100 \%$ dos indivíduos que ainda não usam insulina e até $30 \%$ dos casos, após a operação, mantém apenas o uso dos hipoglicemiantes orais sem a necessidade do uso da insulina?

Apesar da conscientização da população sobre a importância da perda de peso, a obesidade tornou-se nos últimos anos epidemia global. Mais de $80 \%$ dos indivíduos com T2DM são portadores de sobrepeso ou obesidade. A cada $20 \%$ de aumento do peso corporal acima do ideal duplica a probabilidade do paciente se tornar portador de $\mathrm{T}_{2} \mathrm{DM}^{5}$.

É interessante notar que o controle dos níveis glicêmicos ocorre poucos dias após a operação, antes mesmo que ocorra perda de peso significativa; mostrando que a cura do diabetes não está atribuída à redução do peso, tampouco a melhora da resistência insulínica. Na verdade, a intensa redução da ingestão alimentar, acompanhada da paradoxal redução do apetite, é atribuída à redução da produção do hormônio grelina (um orexígeno endógeno) pela exclusão do fundo gástrico do trânsito alimentar ${ }^{13}$.

Com a retirada do fundo gástrico se remove a maior fonte de produção de grelina, o que não ocorria com as operações antigas como a banda gástrica e Scopinaro, já que ambas mantém o fundo gástrico e isso leva a elevação os níveis de grelina.

Buchwald et al. ${ }^{1}$ realizaram metaanálise com 22.094 pacientes com T2DM submetidos as operações dos tipos banda gástrica, bypass gástrico com Y-de-Roux e a derivação biliopancreática, onde foram comparadas as perdas de excesso de peso e a resolução do diabetes após cada uma destas operações. O resultado na banda gástrica foi perda de peso de $47,5 \%$ e resolução do T2DM de 47,9\%; $61,6 \%$ de perda de peso e $83,7 \%$ resolução do T2DM no bypass gástrico e $70,15 \%$ de perda de peso com $98,9 \%$ de remissão do T2DM na derivação biliopancreática. Ainda faltam na literatura trabalhos que comparem a SG com as demais técnicas já utilizadas no controle do T2 $\mathrm{DM}^{7}$.

Existem estudos que vêem a SG como uma operação mista, possuindo o componente restritivo pelo menor volume gástrico (varia de $50 \mathrm{a} 200 \mathrm{~mL}$ ), e o componente entero-hormonal de alteração dos níveis de grelina, GLP1 e PPY. Ao contrário das operações puramente restritivas, a gastroplastia em manga, ao invés de retardar o esvaziamento gástrico e favorecer os vômitos, promove o aceleramento do esvaziamento gástrico e os vômitos são praticamente ausentes ${ }^{1,12}$.

Atualmente o T2DM ainda não faz parte das indicações cirúrgicas em que se dá prioridade para a realização da técnica da gastroplastia em manga, sendo hoje as indicações: super obesos (IMC > 50), obesos com doenças graves associadas, conversão de operação bariátrica prévia (banda gástrica ajustável), decisão no intra-operatório devido a condições adversas como aderências intensas, hepatomegalia extensa, mesentério curto ou com infiltrado gorduroso intenso, instabilidade hemodinâmica; primeiro estágio de duodeno-switch ou Fobi-Capella em pacientes de alto risco; e algumas indicações relativas como: pacientes com risco aumentado de câncer gástrico já que com a SG se evita um estômago excluso, crianças e idosos $>70$ anos, cirrose hepática, pré ou pós transplante renal ou hepático, osteo- 
porose e anemia grave. Neste trabalho verifica-se como a SG atua em relação a T2DM e se pode ser indicada para esta comorbidade específica.

Na SG inicia-se a operações pela dissecção da grande curvatura com a preocupação de liberação de todo o fundo gástrico, evitando deixar reservatório residual de fundo gástrico. Após o estômago estar todo liberado se procede à dissecção junto ao ângulo esôfago-gástrico, identificado a parede esofágica e nesse passo pode se associar, caso seja necessário, a correção de possível hérnia hiatal. A distância do piloro recomendada para o início do grampeamento pode ser $6 \mathrm{~cm}$ em casos que se queira preservar a bomba antral e 2 a $3 \mathrm{~cm}$ em operações mais restritivas. O tamanho da bolsa gástrica é de acordo com a modelagem da sonda de Fouchet, deixando o reservatório gástrico com volume de 30 a 200 $\mathrm{mL}$. Faz-se, então, o grampeamento e secção gástrica paralela a pequena curvatura. A técnica é mais rápida e segura se comparada com outras operações em que se tem de utilizar alça de delgado e maior número de anastomoses, diminuindo o risco de deiscência de anastomose, fístula, e maior aceitabilidade do paciente devido a maiores complicações disabsortivas no pós-operatório ${ }^{2,3,4,8,9}$.
Não existem ainda dados sobre o impacto da cirurgia sobre as complicações crônicas micro e macro vasculares do T2 $\mathrm{DM}^{6}$. Da mesma forma, é ainda incerto se haverá aumento da longevidade dos pacientes operados. Novamente, estudos controlados de longo prazo, como o SOS, deverão fornecer mais respostas para estas questões

Assim como na literatura americana, um dos efeitos esperados para a cirurgia bariátrica é a eficácia na prevenção e cura da T2DM como o observado com os pacientes estudados no HRSJHMG em que se obteve já nos três primeiros meses, cura em $53,33 \%$ dos pacientes e remissão em $46,66 \%$ com $57,14 \%$ dos pacientes mantendo apenas o uso de hipoglicemiantes orais sem a necessidade do uso da insulina.

\section{CONCLUSÃO}

A SG pode ser considerada eficaz no controle do T2DM, necessitando de estudos a longo prazo para que possa ser comparada com as demais técnicas.

Andriani AC, Neves TT. Sleeve gastrectomy and diabetes. ABCD Arq Bras Cir Dig 2008;21(3):133-5

ABSTRACT - Background - The type 2 diabetes mellitus is very frequent among morbid obese patients. It has already been proven that the gastric bypass surgery may cures diabetes mellitus. There are few related studies to sleeve gastroplasty and diabetes. However, promising results exist. Aim - To analyse the glicemic results in patients submmitted to sleeve gastroplasty. Methods - A 11 months prospective study on 15 patients was dome measuring their glycemic levels pre and postoperatively. The basic inclusion criteria was body mass index (BMI) between $35 \mathrm{and} 40 \mathrm{~kg} / \mathrm{m}^{2}$. All patients were operated by sleeve gastroplasty. Results - All 15 patients lowered their glycemic level on their imidiate postoperative period and some achieved normal levels. Conclusions - The sleeve gastroplasty cures diabetes by lowering the plasma glucose and have a long lasting weight loss.

HEADINGS - Gastrectomy. Diabetes mellitus. Obesity, morbid.

\section{REFERÊNCIAS}

1. Buchwald H, Aviador Y, Braunwald E, et al. Bariatric surgery: a systematic review and meta-analysis. J Am Med Assoc 2004; 292: 1724-1737.

2. Cummings DE, Overduim J, Foster-Schubert KE. Gastric bypass for obesity: mechanisms of weight loss and diabetes resolution. J Clin Endocrinol Metab. 2004;89:2608-15.

3. Deitel M, Crosby RD, Gangner M. The First International Consensus Summit for Sleeve Gastrectomy (SG), New York City, 25-27 October 2007. Obes Suurg 2008; 18:487-96.

4. Edward E. Mason, Gastric Emptying Controls Type 2 Diabetes Mellitus. Obesity Surgery, 17, 2007.

5. Francesco Rubino IS. Type 2 Diabetes na Operable Intestinal Disease?A provocative yet reasonable hypothesis. Diabetes Care, Volume 31, Supplement 2, February 2008.

6. Geloneze B, Repetto EM, Pilla VF, Tambascia MA, Pareja JC. Ghrelin a gutbrain hormone. Effect of gastrict bypass. Obes Surg 2003; 13:17-22.

7. Gianfranco Silecchia, Effectiveness of Laparoscopic Sleeve Gastrectomy (Firts Stage of Biliopancreatic Diversion with Duodenal Switch) on Co-Morbibidades in Super-Obese High-Risk Patients. Obesity Sugery, 16, 2006.

8. J. Vidal, Type 2 Diabetes Mellitus and the Metabolic Syndrome Following Sleeve Gastrectomy in Severely Obese Subjects, Obes Surg April 2008.
9. Kral JG, Näsluund E. Surgical treatment of obesity. Nat Clin Pract Endocrinol Metab. 2007;3;574-83.

10. Long SD, O’Brien K, MacDonald KG Jr, Leggett-Frazier N, Swanson MS, Pories $\mathrm{WJ}$, et al. Weigght loss in severely obese subjects prevents the progression of impaired glucose tolerance to type II diabetes. A longitudinal interventional study. Diabetes Care 1994; 17:372-5.

11. Pories WJ, MacDonald KG, Morrgan EJ, et al. Surgical treatment of obesity and its effect on diabetes: 10-y follow-up. Am J Clin Nutr 1992; 55: 582-5S.

12. Rodrigo N. Lamounier, Incretins: Clinical Physiology and Bariatric Surgery Correlating the Entero-endocrine System and a Potentially Anti-dysmetabolic Procedure. Obesity Surgery, 17, 569-576, 2007.

13. Tian Tian Wang, Ileal Transposition Controls Diabetes as Well as Modified Duodenal jejunal Bypass With Better Lipid Lowering in a Nonobese Rat Modelo f Type II Diabetes by Increasing GLP-1. Annals of Surgery, Volume 247, Number 6, June 2008.

14. Tracy-Ann Moo and Francesco Rubino, Gastrointestinal surgery as treatment for type 2 diabetes. Currente Opinion in Endocrinology, Diabetes e Obesity 2008, 15: 153-158.

\footnotetext{
Fonte de financiamento: não há Conflito de interesse: não há Recebido para publicação: 18/03/2008 Aceito para publicação: 11/06/2008
} 